\title{
Two percent ethylenediaminetetraacetic acid chelation treatment for band-shaped keratopathy, without blunt scratching after removal of the corneal epithelium
}

\author{
This article was published in the following Dove Press journal: \\ Clinical Ophthalmology \\ 2 February 2015 \\ Number of times this article has been viewed
}

\author{
Wataru Kobayashi' \\ Shunji Yokokura' \\ Takehiro Hariya' \\ Toru Nakazawa ${ }^{1-3}$ \\ 'Department of Ophthalmology, \\ ${ }^{2}$ Department of Retinal Disease \\ Control, Ophthalmology, ${ }^{3}$ Department \\ of Advanced Ophthalmic Medicine, \\ Tohoku University Graduate School \\ of Medicine, Sendai, Japan
}

Background: The purpose of this study was to assess the effectiveness of $2 \%$ ethylenediaminetetraacetic acid (EDTA) for the treatment of band-shaped keratopathy.

Methods: We studied 24 eyes of 16 patients with band-shaped keratopathy who underwent EDTA chelation treatment from April 1, 2011 to December 31, 2012. We compared preoperative and 1 month postoperative logarithm of the minimum angle of resolution (logMAR) best corrected visual acuity, intraocular pressure, and corneal curvature radius (K1, horizontal meridian; K2, vertical meridian; Km, average of K1 and K2). The Mann-Whitney $U$-test was used to determine the significance of differences.

Results: There was a significant difference in preoperative and postoperative logMAR best corrected visual acuity $(P=0.01)$. There were no significant differences in preoperative and postoperative intraocular pressure $(P=0.24)$ or corneal curvature radius $(\mathrm{K} 1, P=0.54 ; \mathrm{K} 2$, $P=0.49 ; \mathrm{Km}, P=0.45)$.

Conclusion: After 2\% EDTA chelation treatment, post-operative logMAR best corrected visual acuity improved significantly. Moreover, since there was no significant difference in corneal curvature radius, there was little influence on corneal surface form. We believe that the results of our $2 \%$ EDTA chelation treatment were comparable with results obtained with $3.75 \%$ EDTA chelation treatment in previous reports. Two percent EDTA chelation is an effective treatment for band-shaped keratopathy and a useful method for any institution.

Keywords: ethylenediaminetetraacetic acid, band-shaped keratopathy, phototherapeutic keratectomy, cornea

\section{Introduction}

Band-shaped keratopathy (BSK) is characterized by the deposition of a whitish, grayish, or yellowish opacity in the superficial layers of the central cornea. In the early stages, it appears in the nasal or temporal margins of the cornea, leaving a clear space between the corneal limbus and the opacity. The opacity then gradually extends to the central part of the cornea and becomes band-shaped. Histopathologically, such opacities are composed of calcium salt deposited in Bowman's membrane. The calcium salt depositions may sometimes progress to the parenchyma of the cornea, just under Bowman's membrane, ${ }^{1,2}$ and Bowman's membrane itself may also sometimes rupture. ${ }^{3}$

The pathogenesis of BSK is sometimes associated with ophthalmopathies, such as deep keratitis, glaucoma, and uveitis, with eyes injected with silicone oil, with traumatic corneal disorders, and with systemic illnesses, such as chronic renal failure, hyperparathyroidism, and sarcoidosis; it may also be idiopathic. ${ }^{1,4-9}$ Tears and the aqueous
Correspondence: Shunji Yokokura Department of Ophthalmology, Tohoku University Graduate School of Medicine, I-I Seiryo-cho, Aoba-ku, Sendai, Miyagi 980-8574, Japan

Tel +81227177294

Fax +8I 227177298

Email yokokura@oph.med.tohoku.ac.jp 
humor contain calcium in concentrations that approach their solubility product. It is thought that the calcification in BSK is caused by elevated surface $\mathrm{pH}$, an increase in the evaporation of tears, or increased calcium concentration accompanying inflammation, but the precise mechanism has not been identified. ${ }^{2,10}$

Patients suffering from BSK may complain of problems such as decreased vision, bleary eyes, ocular irritation, and sensations of an ocular foreign body. Treatments for BSK include mechanical scratching, application of hydrochloric acid, ${ }^{11,12}$ lamellar keratoplasty, ${ }^{13}$ phototherapeutic keratectomy (PTK), ${ }^{14,15}$ and chelation therapy using ethylenediaminetetraacetic acid (EDTA), ${ }^{12,16-19}$ among others.

EDTA is a chelating agent, the original uses of which were mainly in dialysis. EDTA always reacts with a ratio of $1: 1$, regardless of the electric charge of the reacting metal ions, and produces a colorless and water-soluble chelate. EDTA-Na ${ }_{2}$ solution is nearly neutral and removes deposited calcium salt molecules with a physical chelating effect. PTK treatment for BSK is widely performed, but treatment facilities are limited because of the high cost of the excimer laser system. Additionally, BSK may recur, and it is difficult to repeatedly treat the same eye with PTK.

EDTA-Na $a_{2}$ chelation treatment for BSK has previously been reported, but the concentration of EDTA that was studied has varied, ranging from $1.7 \%$ to $3.75 \%,{ }^{16,17,20}$ and a consistent standard has not been established. Moreover, in previous reports, blunt scratching of the opacity was necessary after removal of the corneal epithelium. ${ }^{16,21}$ Our method, by contrast, does not require blunt scratching after removal of the corneal epithelium, uses 2\% EDTA, and has given good operational results. This report describes the procedure and our results.

\section{Subjects and methods Inclusion criteria}

This retrospective, cross-sectional study comprised a total of 24 eyes in 16 Japanese adult patients with BSK. A summary of the clinical data is shown in Table 1. All the patients had blurred vision caused by BSK. The inclusion criterion was a diagnosis of BSK by slit-lamp microscopy. The exclusion criterion was uncontrolled concomitant eye disease (other than cataracts), including infectious keratitis, traumatic corneal disorder, uveitis, macular disease, and optic nerve disease. The baseline clinical parameters recorded for each patient were age, sex, corneal curvature radius, the logarithm of the minimum angle of resolution ( $\log$ MAR) best corrected visual acuity (BCVA), and intraocular pressure (IOP). The corneal curvature radius was measured with an auto refracto/keratometer
Table I Patient characteristics and causes of BSK

\begin{tabular}{lllll}
\hline Characteristics & $\begin{array}{l}\text { Number, } \\
\text { mean } \pm \text { SD }\end{array}$ & Cause & Eyes, $\mathbf{n} \%$ \\
\hline Age (years) & $71.7 \pm 15.0$ & Idiopathic & 13 & 54.2 \\
Sex & & Chronic renal failure & $5^{*}$ & 20.8 \\
$\quad$ Male & 7 (9 eyes) & Diabetes mellitus & $8^{*}$ & 33.3 \\
Female & 9 (I5 eyes) & Hypercalcemia & 0 & 0 \\
Laterality & & Hyperparathyroidism & 0 & 0 \\
$\quad$ Right & II & Sarcoidosis & 0 & 0 \\
$\quad$ Left & 13 & & & \\
\hline
\end{tabular}

Note: *Two eyes had both chronic renal failure and diabetes mellitus. Abbreviations: BSK, band-shaped keratopathy; SD, standard deviation.

(RKT-7700, Nidek Inc, Fremont, CA, USA). Baseline BCVA was measured with a standard Japanese decimal visual acuity chart, and converted to logMAR for statistical analysis. IOP was measured with a noncontact tonometer (NT-4000, Nidek Inc) at the time of initial diagnosis of BSK. When measurable, corneal endothelial cells were measured with noncontact specular microscopy (SP-3000P, Topcon Medical Laser Systems, Inc, Santa Clara, CA, USA). The study adhered to the tenets of the Declaration of Helsinki, and the protocols were approved by the clinical research ethics committee of the Tohoku University Graduate School of Medicine.

\section{Surgical technique}

We performed instillation anesthesia (with Xylocaine) on the eye undergoing the procedure, and used a lid speculum to hold the eyelid open. We determined the extent of opacity with the scleral scattering method, in which an endoilluminator, commonly used in 20-gage vitrectomy, is placed on the limbus of the cornea. We then carefully removed the corneal epithelium from the area of the opacity with a golf club spud (Figure 1A) and applied a fragment of EDTA-dipped surgical sponge (MQA, Inami, Tokyo, Japan) to the opacity for 5 minutes (Figure 1B). We next removed the MQA fragment, and checked for any remaining opacity with the light guide (Figure 1C). If the opacity was not completely removed, we applied the MQA fragment for another 5 minutes. We repeated this procedure as necessary. Finally, after the opacity was completely removed, we washed the eye surface with approximately $50 \mathrm{~mL}$ of normal saline, put a contact lens on the eye, and applied an antibiotic ointment. After the operation, the patient performed antibiotic and steroid instillation four times daily and wore a contact lens until epithelialization of the cornea was complete. All patients were examined on the day following the operation and then discharged. Follow-up examinations were performed 1 week and 1 month after the operation, and at various other intervals depending on the case. 

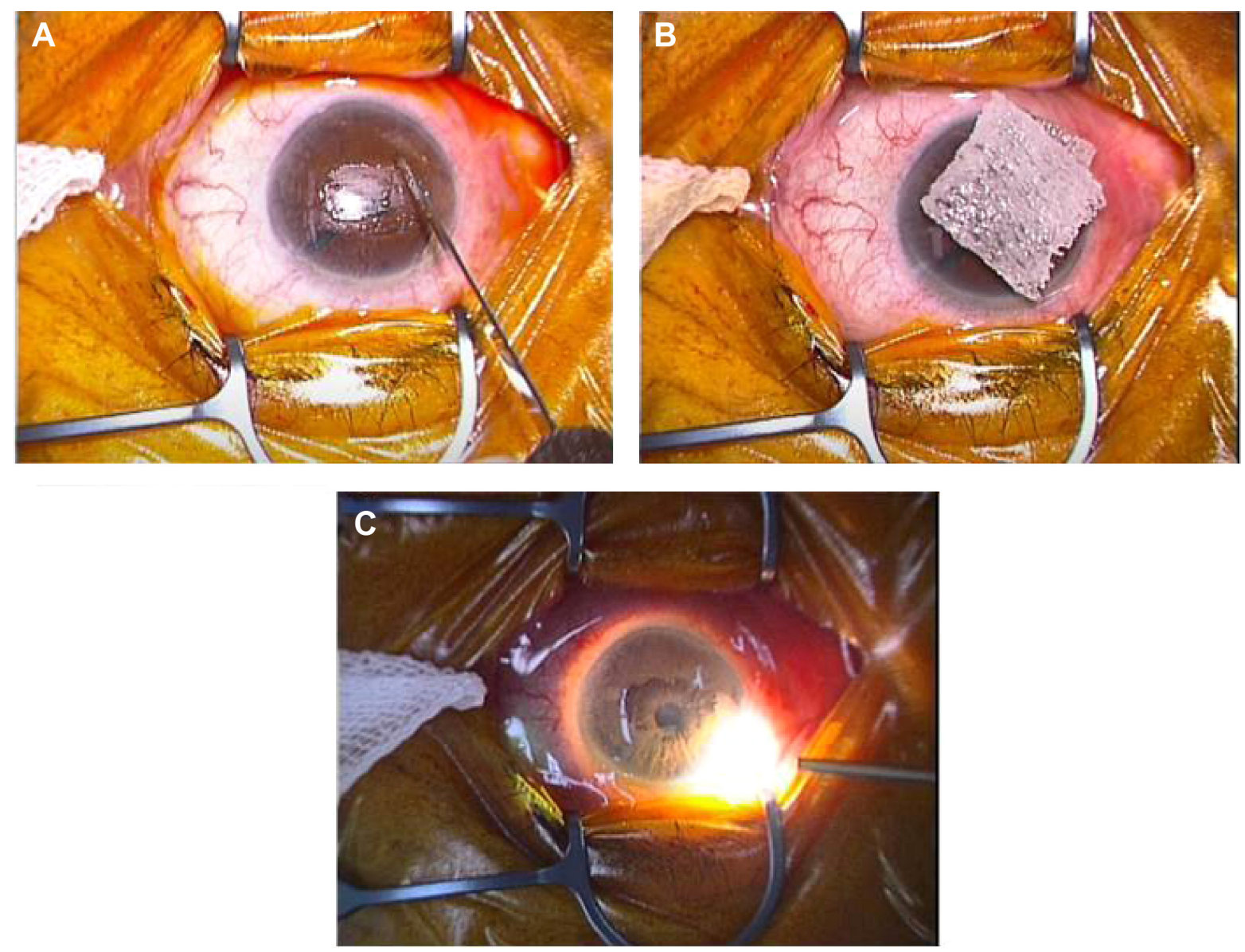

Figure I Surgical technique.

Notes: (A) Removal of the corneal epithelium from the area of the opacity with a golf club spud. (B) Applying a fragment of MQA (surgical sponge) dipped in EDTA to the opacity. (C) Checking for any remaining opacity with a light guide.

Abbreviation: EDTA, ethylenediaminetetraacetic acid.

The 2\% EDTA solution (pH circa 6.0, 348 osmol) comprised $1 \mathrm{~g}$ of EDTA $\left(\mathrm{Na}_{2}\right), 0.56 \mathrm{~g}$ of $\mathrm{NaCl}$, and $0.17 \mathrm{~g}$ of $\mathrm{Na}_{2} \mathrm{CO}_{3}$, with distilled water for injection added to obtain $50 \mathrm{~mL}$ of solution. We performed mechanical sterilization with a $0.22 \mu \mathrm{m}$ filter on a clean bench and then placed the solution into a purified eyedropper. The reagents and other materials used were EDTA/2Na (Dojindo), sodium carbonate and sodium chloride (Wako Pure Chemicals), distilled water $(20 \mathrm{~mL} \times 3$; Otsuka Pharmaceutical Factory, Inc, Tokushima, Japan), a Millex-GV syringe driven filter unit (Millipore, Billerica, MA, USA), and a $50 \mathrm{~mL}$ syringe.

\section{Statistical analysis}

The Mann-Whitney $U$-test was used to determine the significance of preoperative and postoperative differences in $\log$ MAR BCVA, corneal curvature radius, IOP, corneal endothelial cells (per $\mathrm{mm}^{2}$ ), coefficient of variation $(\mathrm{CV})$, and the percentage of hexagonal cells (\%HEX). Statistical analysis was carried out with GraphPad Prism version 6
(GraphPad Software, San Diego, CA, USA). $P$-values $<0.05$ were considered to be statistically significant.

\section{Results}

A summary of our results is shown in Table 2. The total number of patients in this study was 16 (24 eyes). The ratio of men to women was 7:9 (9:15 eyes), and the ratio of left to right eyes was 13:11.

The mean patient age was $71.7 \pm 15.0$ years. The mean preoperative $\log \mathrm{MAR} B C V A$ was $0.69 \pm 0.76$, the mean preoperative corneal curvature radii were $7.81 \pm 0.34,7.54 \pm 0.33$, and $7.68 \pm 0.31$ (K1, K2, and $\mathrm{Km}$, respectively) and the mean preoperative IOP was $15.2 \pm 4.4 \mathrm{mmHg}$. The mean postoperative $\log$ MAR BCVA was $0.41 \pm 0.67$, the mean postoperative corneal curvature radii were $7.80 \pm 0.34,7.67 \pm 0.31$, and $7.66 \pm 0.33$ (K1, K2, and $\mathrm{Km}$, respectively), and the mean postoperative IOP was $14.1 \pm 4.0 \mathrm{mmHg}$.

There was a significant difference between preoperative and postoperative $\log$ MAR BCVA $(P=0.01)$, with 

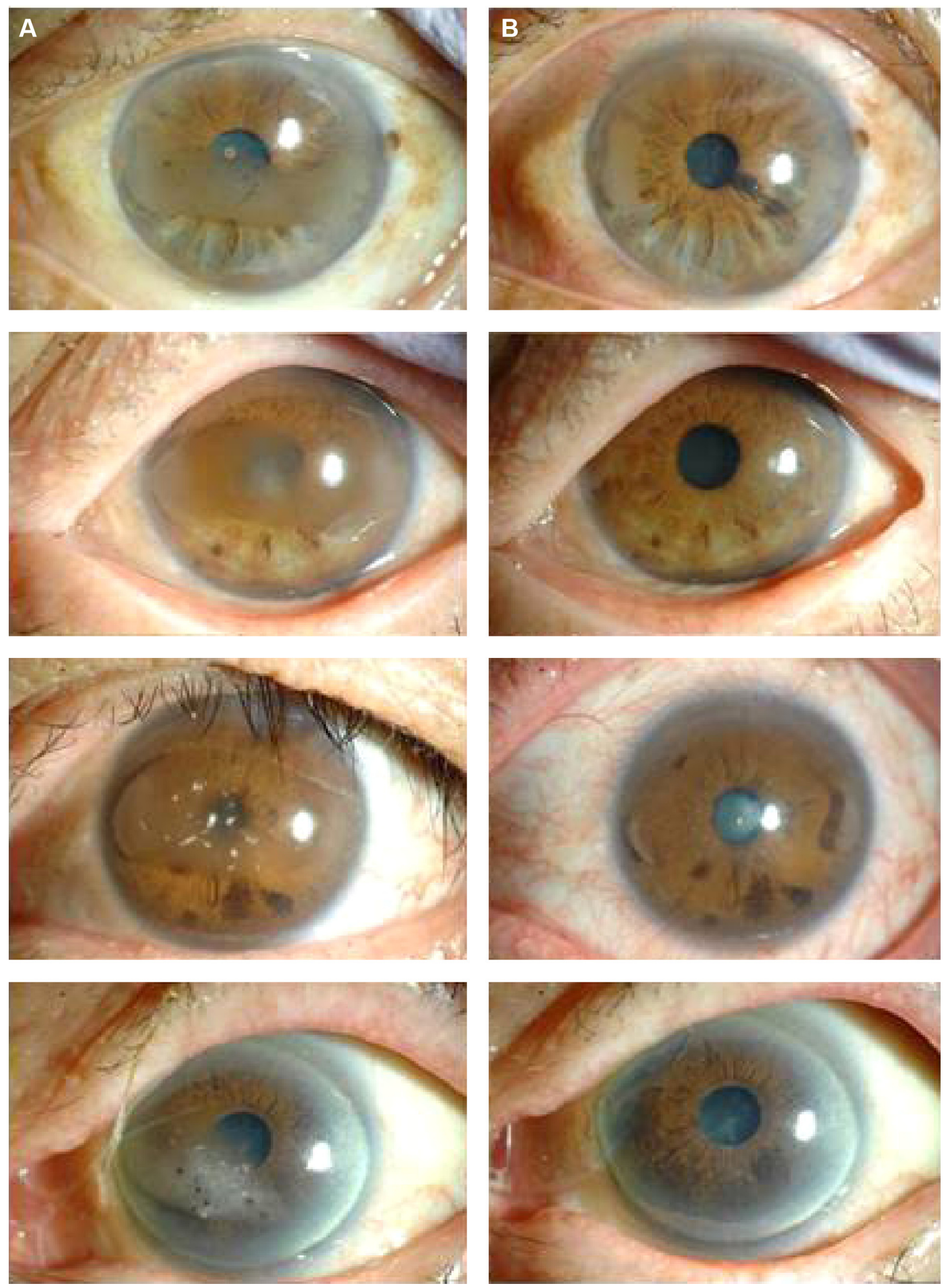

Figure 2 Representative images of eyes with BSK before (A) and after (B) EDTA chelation. The patient's symptoms resolved completely after the procedure. Abbreviations: BSK, band-shaped keratopathy; EDTA, ethylenediaminetetraacetic acid. 
Table 2 Pre- and post-operative characteristics

\begin{tabular}{|c|c|c|c|}
\hline Characteristics & Pre-operative & Post-operative & $P$-value \\
\hline Mean BCVA (logMAR) & $0.69 \pm 0.76$ & $0.4 I \pm 0.67$ & $<0.01 *$ \\
\hline \multicolumn{4}{|l|}{ Corneal curvature radius } \\
\hline $\mathrm{KI}$ & $7.8 I \pm 0.34$ & $7.80 \pm 0.34$ & 0.54 \\
\hline $\mathrm{K} 2$ & $7.54 \pm 0.33$ & $7.67 \pm 0.31$ & 0.49 \\
\hline $\mathrm{Km}$ & $7.68 \pm 0.31$ & $7.66 \pm 0.33$ & 0.45 \\
\hline $\mathrm{IOP}(\mathrm{mmHg})$ & $15.2 \pm 4.4$ & $\mid 4.1 \pm 4.0$ & 0.24 \\
\hline Corneal endothelial cells (per $\mathrm{mm}^{2} ; 10$ eyes) & $2,342.2 \pm 985.6$ & $2,250.2 \pm 749.0$ & 0.95 \\
\hline CV (8 eyes) & $29.1 \pm 6.7$ & $27.8 \pm 7.3$ & 0.58 \\
\hline Hexagonal cells (\%HEX; per mm²; 8 eyes) & $47.5 \pm 14.7$ & $61.4 \pm 8.0$ & $<0.05^{*}$ \\
\hline
\end{tabular}

an overall improvement in mean postoperative logMAR BCVA. In all eyes, there was partial or complete amelioration of symptoms after treatment with EDTA (Figure 2). There was no significant difference between preoperative and postoperative corneal curvature radii (K1, K2, and $\mathrm{Km} ; \mathrm{K} 1, P=0.54 ; \mathrm{K} 2, P=0.49 ; \mathrm{Km}, P=0.45)$ or IOP $(P=0.24)$.

In the ten eyes in which corneal endothelial cells (per $\mathrm{mm}^{2}$ ) were detectable, there was no significant postoperative change in the number of corneal endothelial cells $(P=0.95)$. In the eight eyes in which $\mathrm{CV}$ and \%HEX were measurable, there was no significant postoperative change in $\mathrm{CV}$ $(P=0.58)$, but there was a significant postoperative increase in $\% \operatorname{HEX}(P=0.03)$.

\section{Discussion}

Our 2\% EDTA chelation treatment for BSK, in which we applied a fragment of EDTA-dipped MQA after removal of the corneal epithelium, led to positive logMAR BCVA outcomes, and did not lead to any significant change in corneal curvature radius.

Blunt scratching has been used in many previous studies of EDTA treatment. When low-concentration EDTA or hydrochloric acid are used to remove opacities, we believe that the need for blunt scratching can arise because not enough of the opacity has been removed, or because the EDTA has only been applied to the opacity itself. This may lead not only to incomplete removal of the opacity, but also to postoperative irregular astigmatism and decreased visual acuity. In our procedure, scratching was never necessary, and the fragment of MQA dipped in highly concentrated EDTA was only applied to the opacity after removal of the corneal epithelium. We believe that this is why our procedure had little effect on the configuration of the corneal surface. We also believe that the improvement in visual acuity and $\% \mathrm{HEX}$ may be related to this minimization of the effect on the ocular surface, as well as to the actual removal of the opacity.

Recently, the usefulness of PTK treatment for BSK has been established, and it has become a common treatment. ${ }^{14,15,22,23}$ We believe that PTK is a suitable treatment for BSK in institutions equipped with excimer lasers. However, PTK often causes postoperative hypermetropia, ${ }^{14,15}$ for which touch-up laser treatment may be needed. Moreover, it is difficult to precisely calculate intraocular lens power for cataract surgery after PTK, and some accidental errors may occur. ${ }^{24}$ Although our EDTA treatment method requires removal of the corneal epithelium, it does not significantly change the shape of the cornea, leading us to believe that it should be usable even in cases with a history of cataract surgery. In regards to postoperative pain and discomfort, sharp pain was common until 1 week after the operation, when we observed very little sharp pain. Complete epithelialization was observed in all cases. These results were comparable with past reports on PTK treatment.

LogMAR BCVA in eight eyes (33\%) was unchanged, and 1 month after the operation, improved by two or more lines of logMAR BCVA in 12 eyes (50\%). These results were comparable with a past report (Table 3$).{ }^{16}$ We did not note any improvement in visual acuity, possibly due to the strong influence of cataracts, controlled diabetic maculopathy, or the inclusion of patients with good pre-existing visual acuity. Patients with good preexisting visual acuity did, however, show a reduction in blurred vision. The severity of the patients' cataracts was similar before and after the operation. O'Brart et al have reported that mean Snellen visual acuity improved in $88 \%$ of eyes after PTK. ${ }^{14}$ However, O'Brart et al did not classify visual improvement into number of lines on the Snellen chart. ${ }^{14}$ In addition, Najjar et al have reported that 1 month after BSK treatment with 3.75\% EDTA, 33.3\% 
Table 3 Visual outcomes after EDTA treatment

\begin{tabular}{|c|c|c|c|c|c|c|}
\hline \multicolumn{7}{|c|}{ Visual acuity at I-month follow-up (number of eyes) } \\
\hline Initial visual acuity & $<20 / 400$ & $20 / 400$ to $20 / 200$ & $20 / / 100$ to $20 / 50$ & $\geq 20 / 40$ & Total & $\%$ \\
\hline$<20 / 400$ & 1 & 0 & 0 & 0 & 1 & 4.2 \\
\hline $20 / 400$ to $20 / 200$ & I & I & 0 & 0 & 2 & 8.3 \\
\hline $20 / 100$ to $20 / 50$ & I & I & 2 & 0 & 4 & 16.7 \\
\hline$\geq 20 / 40$ & I & 0 & 8 & 8 & 17 & 70.8 \\
\hline
\end{tabular}

Number of lines of visual improvement $(+)$ or worsening $(-)$ compared with a previous report

\begin{tabular}{lllll}
\hline & $-\mathbf{2}$ & $-\mathbf{I}, \mathbf{0},+\mathbf{I}$ & $+\mathbf{2 , 3}$ & $+\mathbf{4}$ \\
\hline $2 \%$ EDTA treatment & $0 \%$ & $50 \%$ & $29.2 \%$ & $20.8 \%$ \\
\hline
\end{tabular}

Abbreviation: EDTA, ethylenediaminetetraacetic acid.

of patients improved by two or more lines on the Snellen chart, with $35.3 \%$ showing the same improvement at the last follow-up visit. ${ }^{16}$ We used a lower concentration of EDTA (2\%) and obtained equivalent results. We believe that this makes our method safer than past EDTA treatment methods.

Although it is known that there can be slight penetration of EDTA into the corneal endothelium, ${ }^{25}$ we believe that this side effect can be minimized by lowering embrocation time and performing sufficient lavage. However, we believe that when there are low numbers of corneal endothelium cells, our technique should be used with caution. There were no complications 1 month after the operation in any of our cases, nor in the nine cases that we followed for at least 3 months. Longer follow-up times may be necessary to obtain sufficient data on recurrence, corneal epithelium disorders, and infections.

\section{Conclusion}

We found that 2\% EDTA chelation treatment for BSK, with EDTA-dipped fragments of MQA applied solely to the cornea, was a useful and promising technique, mainly because of its minimal effect on the corneal surface.

\section{Disclosure}

The authors have no commercial relationships relevant to this work.

\section{References}

1. Jhanji V, Rapuano CJ, Vajpayee RB. Corneal calcific band keratopathy. Curr Opin Ophthalmol. 2011;22(4):283-289.

2. O'Connor GR. Calcific band keratopathy. Trans Am Ophthalmol Soc. 1972;70:58-81.

3. Font R, Green WR, Howes E, Jakobiec FA, Zimmerman L. Ophthalmic Pathology: An Atlas and Textbook. Volume 1. 3rd ed. Philadelphia, PA, USA: WB Saunders; 1985.
4. Kennedy RE, Roca PD, Landers PH. Atypical band keratopathy in glaucoma patients. Trans Am Ophthalmol Soc. 1971;69:124-139.

5. Daniel E, Pistilli M, Pujari SS, et al. Risk of hypotony in noninfectious uveitis. Ophthalmology. 2012;119(11):2377-2385.

6. Sternberg P, Hatchell DL, Foulks GN, Landers MB 3rd. The effect of silicone oil on the cornea. Arch Ophthalmol. 1985;103(1):90-94.

7. Porter R, Crombie AL. Corneal and conjunctival calcification in chronic renal failure. Br J Ophthalmol. 1973;57(5):339-343.

8. Golan A, Savir H, Bar-Meir S, Oliver I, De Vries A. Band keratopathy due to hyperparathyroidism. Ophthalmologica. 1975;171(2):119-122.

9. Johnston RL, Stanford MR, Verma S, Green WT, Graham EM. Resolution of calcific band keratopathy after lowering elevated serum-calcium in a patient with sarcoidosis. Br J Ophthalmol. 1995;79(11):1050-1050.

10. Cogan DG, Albright F, Bartter FC. Hypercalcemia and band keratopathy - report of 19 cases. Arch Ophthalmol. 1948;40(6):624-638.

11. Linhart RW. Treatment of calcareous film of the cornea. Am J Ophthalmol. 1952;35(10):1497-1498.

12. Breinin GM, Devoe AG. Chelation of calcium with edathamil calciumdisodium in band keratopathy and corneal calcium affections. $A M A$ Arch Ophthalmol. 1954;52(6):846-851.

13. Burillon C, Durand L, Berne E, et al. [Semiological value of 23 cases of band keratopathy]. J Fr Ophtalmol. 1992;15(11):579-586. French.

14. O'Brart DP, Gartry DS, Lohmann CP, Patmore AL, Kerr Muir MG, Marshall J. Treatment of band keratopathy by excimer-laser phototherapeutic keratectomy - surgical techniques and long-term follow-up. Br J Ophthalmol. 1993;77(11):702-708.

15. Stewart OG, Morrell AJ. Management of band keratopathy with excimer phototherapeutic keratectomy: visual, refractive, and symptomatic outcome. Eye. 2003;17(2):233-237.

16. Najjar DM, Cohen EJ, Rapuano CJ, Laibson PR. EDTA chelation for calcific band keratopathy: results and long-term follow-up. Am J Ophthalmol. 2004;137(6):1056-1064.

17. Grant WM. New treatment for calcific corneal opacities. AMA Arch Ophthalmol. 1952;48(6):681-685.

18. Scherz W, Vogel M. [Treatment of band keratopathy with EDTA (author's transl)]. Klin Monbl Augenheilkd. 1978;172(3):371-378. German.

19. Alexandridis A, Stefani FH. [Treatment of band keratopathy (author's trans1)]. Klin Monbl Augenheilkd. 1980;176(6):968-971. German.

20. Lam HY, Wiggs JL, Jurkunas UV. Unusual presentation of presumed posterior polymorphous dystrophy associated with iris heterochromia, band keratopathy, and keratoconus. Cornea. 2010;29(10):1180-1185.

21. Im S-K, Lee K-H, Yoon K-C. Combined ethylenediaminetetraacetic acid chelation, phototherapeutic keratectomy and amniotic membrane transplantation for treatment of band keratopathy. Korean JOphthalmol. 2010;24(2):73-77. 
22. Campos M, Nielsen S, Szerenyi K, Garbus JJ, McDonnell PJ. Clinical follow-up of phototherapeutic keratectomy for treatment of corneal opacities. Am J Ophthalmol. 1993;115(4):433-440.

23. Gartry D, Muir MK, Marshall J. Excimer laser treatment of corneal surface pathology - a laboratory and clinical-study. Br J Ophthalmol. 1991;75(5):258-269.
24. Seitz B, Langenbucher A, Nguyen NX, et al. Underestimation of intraocular lens power for cataract surgery after myopic photorefractive keratectomy. Ophthalmology. 1999;106(4):693-702.

25. López Bernal D, Ubels JL. Quantitative evaluation of the corneal epithelial barrier: effect of artificial tears and preservatives. Curr Res Eye. 1991;10(7):P645-P656.

\section{Publish your work in this journal}

Clinical Ophthalmology is an international, peer-reviewed journal covering all subspecialties within ophthalmology. Key topics include: Optometry; Visual science; Pharmacology and drug therapy in eye diseases; Basic Sciences; Primary and Secondary eye care; Patient Safety and Quality of Care Improvements. This journal is indexed on

Submit your manuscript here: http://www.dovepress.com/clinical-ophthalmology-journal
Dovepress

PubMed Central and CAS, and is the official journal of The Society of Clinical Ophthalmology (SCO). The manuscript management system is completely online and includes a very quick and fair peer-review system, which is all easy to use. Visit http://www.dovepress.com/ testimonials.php to read real quotes from published authors. 